\title{
Une révolution à l'œuvre : le faubourg Saint-Marcel
} (1789-1794)

Exposé de soutenance de thèse d'État

\section{Haim Burstin}

\section{(2) OpenEdition \\ Journals}

Édition électronique

URL : https://journals.openedition.org/ahrf/1827

DOI : 10.4000/ahrf.1827

ISSN : 1952-403X

Éditeur :

Armand Colin, Société des études robespierristes

Édition imprimée

Date de publication : 1 mars 2001

Pagination : 93-101

ISSN : 0003-4436

Référence électronique

Haim Burstin, « Une révolution à l'œuvre : le faubourg Saint-Marcel (1789-1794) », Annales historiques de la Révolution française [En ligne], 323 | janvier-mars 2001, mis en ligne le 16 mars 2006, consulté le 23 avril 2022. URL : http://journals.openedition.org/ahrf/1827 ; DOI : https://doi.org/10.4000/ahrf. 1827

Ce document a été généré automatiquement le 23 avril 2022.

Tous droits réservés 


\title{
Une révolution à l'œuvre : le faubourg Saint-Marcel (1789-1794)
}

Exposé de soutenance de thèse d'État ${ }^{1}$

\author{
Haim Burstin
}

1 Le projet initial de la thèse que je présente aujourd'hui plonge ses racines dans une saison historiographique particulièrement heureuse pour les études sur Paris révolutionnaire celle qui se situe entre les années 50 et 70 . À cette époque arrivaient à maturation les travaux d'Albert Soboul, Richard Cobb, Georges Rudé, Walter Markov, Käre Tønnesson, Marcel Reinhart une génération d'historiens dont les efforts convergeaient pour donner plein éclat à ce secteur d'études. Si l'on songe que ce grand renouveau de l'histoire parisienne s'enchaînait, vers la fin des années 60 , avec une vague de contestation sociale et politique de grande ampleur, caractérisée par d'importantes émeutes urbaines, on comprend qu'il y avait de quoi passionner le jeune chercheur que j'étais je trouvais dans cet ensemble de circonstances une incitation à chercher des précédents illustres de ce que je venais de voir se passer sous mes yeux.

2 Néanmoins l'extraordinaire richesse des études qui venaient de paraître semblait décourager de nouvelles recherches en cette direction : l'impression était que, vu l'état des sources, l'étude était pour l'essentiel achevée et qu'il ne restait qu'à glaner. La preuve en est que ce secteur fut progressivement délaissé et l'intérêt des historiens se porta vers d'autres sujets. D'autant plus que les archives parisiennes, comme tout le monde sait, sont caractérisées par de profondes lacunes provoquées par les incendies de 1871, ce qui compliquait davantage les choses.

3 Le projet de pousser plus loin mes connaissances sur Paris, malgré tous ces inconvénients, prenait donc le goût d'un défi. Un défi que j'avais décidé de relever dès le début de mes recherches en France, dans le cadre de l'Institut d'histoire de la Révolution française - mon premier point de chute à Paris - où Albert Soboul, dont j'ai le plaisir d'évoquer ici le souvenir, venait de créer un séminaire de doctorat.

4 Mais encore fallait-il savoir quel chemin emprunter sur un terrain -celui de la Révolution parisienne - qui s'avérait sans doute parmi les plus battus de l'histoire de France. Le premier critère adopté fut celui d'une réduction d'échelle, par le choix 
d'étudier un seul quartier, le faubourg Saint-Marcel, renommé pour sa pauvreté et pour son tempérament révolutionnaire ; ce qui revenait à rouvrir le dossier sur les couches populaires parisiennes. À ce niveau toutefois, le problème des sources se compliquait davantage, imposant un changement de stratégie: abandonner donc provisoirement toute tentative de reconstruire une histoire politique sur la base d'une documentation trop fragmentaire, pour emprunter un chemin détourné visant à approfondir d'abord la connaissance du faubourg Saint-Marcel par une étude de sa physionomie démographique, économique et sociale.

Ce premier travail donnait lieu à une thèse de $3 \mathrm{e}$ cycle entièrement consacrée à ces questions $\left({ }^{2}\right)$; les résultats de cette étude, en enrichissant ma connaissance du vieux faubourg, me permettaient d'aborder son histoire proprement politique en faisant appel à un éventail thématique large, fondé sur un ensemble de sources plus riche et varié que celui que j'avais initialement envisagé. La documentation n'était pas pour autant moins lacunaire, mais les pistes à suivre s'en trouvaient multipliées, notamment par la possibilité de faire parler des témoignages liés à la vie profonde du quartier, et de les relier à l'événement révolutionnaire sans une telle étude préalable plusieurs de ces témoignages seraient restés muets ou insignifiants.

D'ailleurs le rapport entre figures sociales et comportements politiques demeure une question encore insuffisamment explorée dans le cadre parisien: on ne peut pas, par exemple, déduire l'artisan du sans-culotte, comme souvent il a été fait il faut au contraire essayer de comprendre comment et par quel processus l'artisan devient un sans-culotte. Même si sous la Révolution la politique tend à envahir le quotidien de la population parisienne et à s'y superposer, les gens ne cessent pas pour autant de vivre et de travailler dans le cadre traditionnel de leur quartier et de faire face à leurs problèmes concrets. Mais réciproquement il serait naïf, comme souvent on le prétend, de voir le monde de l'atelier et de la boutique confluer automatiquement dans celui du sans-culotte il s'agit au contraire d'un complexe métabolisme, fait de continuités et de ruptures, que j'ai essayé d'observer de près. Social et politique se révèlent donc strictement imbriqués et la question sociale rentre à part entière à composer ce que j'ai appelé l'imaginaire politique populaire, à savoir le système d'attentes qu'au niveau populaire on confie à la politique.

7 Après une étude autant fouillée que possible sur les caractères du faubourg SaintMarcel vers la fin du xviIIe siècle, je pouvais revenir finalement à son histoire politique armé cette fois-ci d'une grille élargie et d'un éventail thématique plus riche, ce qui me permettait d'augmenter les sources à interpeller. Ceci dans une stratégie de recherche qui prévoyait d'une part une dilatation chronologique sur toute la période comprise entre 1789 et Thermidor et, d'autre part, le projet de rassembler tous azimuts autant de données possibles ayant affaire à l'histoire du faubourg, sans négliger celles qui, à première vue, n'apparaissaient pas immédiatement éclairantes. Il fallait donc affronter jusqu'aux détails dans le but non pas d'une miniature, mais d'une grande fresque où rassembler une masse documentaire unique par sa taille et sa qualité. Le dossier s'avérait ainsi fort épais, néanmoins il ne s'agissait pas d'un simple travail d'accumulation mais d'un expédient heuristique pour faire face aux lacunes documentaires; le défi se déplaçait et s'affinait car il s'agissait d'essayer d'incorporer par agglutination une masse des matériaux disparates pour en faire un ensemble cohérent. 
8 Certes cette technique et le choix de la petite échelle comportaient des inconvénients notamment le risque d'en arriver, par souci d'exhaustivité, au microscopique. Cependant je crois que ce type d'approche en soi sollicite implicitement un changement dans la qualité des attentes non pas une synthèse, mais la possibilité de se mettre à l'écoute des différents segments de la société révolutionnaire en les laissant parler pour saisir toute sa créativité et sa capacité d'élaboration politique : voilà un laboratoire en mouvement qui donne lieu à une gamme très variée de comportements politiques.

D'ailleurs, si le choix a été celui de la petite échelle, encore faut-il réfléchir sur le caractère de cette échelle les quatre sections du faubourg Saint-Marcel représentaient tout de même 1/10 de la population parisienne, donc environ 60000 habitants, ce qui correspond à une ville moyenne d'Ancien Régime. En plus il n'a pas été adopté un registre univoque, mais une sorte d'alternance entre différentes échelles: le champ d'observation peut se réduire du quartier à l'îlot et même aux individus avec leurs biographies en revanche il peut s'élargir à des réalités plus étendues, comme le bloc des faubourgs populaires ou la ville dans son ensemble, lorsqu'une démarche comparative s'impose. La modulation entre différentes échelles est régie par l'état des sources aussi bien que par le souffle de la conjoncture faubourienne: lorsque le quartier tend à se déployer, s'orientant vers la ville dans son ensemble pour en partager l'histoire, l'échelle augmente lorsqu'au contraire le quartier tend à se replier sur lui-même et sur ses problèmes spécifiques, le champ d'observation doit forcément se restreindre. À ce sujet il serait important de savoir si ce mouvement s'inscrit dans un rythme synchronique partagé par d'autres quartiers parisiens.

10 En adoptant ce procédé il est inévitable de se laisser piloter par les sources celles-ci tendent à se rassembler spontanément autour de certains moments forts de la conjoncture faubourienne, mais aussi autour de certains noyaux thématiques que j'ai suivis tout au cours de cette recherche, au prix d'un très long et patient travail de montage. Néanmoins, si une bonne partie des documents recueillis se reliaient entre eux grâce à une sorte de tissu conjonctif naturel, pour une importante partie des données rassemblées à la suite de la multiplication des paramètres de recherche, il a fallu avoir recours à une technique en quelque sorte " pointilliste ". Autrement dit, là où les renseignements tirés des archives ne s'intégraient pas spontanément, ils ont été assemblés par juxtaposition c'est souvent cette juxtaposition qui permet la composition d'une image que, sans cela, il serait difficile de saisir. Voilà donc ce qui, plus qu'un choix, s'est révélé un critère pour essayer de compenser les lacunes présentes au niveau documentaire.

On pourrait voir dans cette méthode un certain abus d'empirisme. Une démarche comme la nôtre, essentiellement pragmatique, qui plaide pour un retour aux sources et prétend leur laisser la parole, privilégiant les archives aux travaux, s'inscrit en effet en contrepoint par rapport notamment à une tradition consolidée dans l'historiographie politique de la Révolution caractérisée par un fort encadrement idéologique et catégoriel ou par de fortes ambitions conceptualisantes. Cependant, une telle tradition risque de court-circuiter en quelque sorte la recherche, en faisant de la Révolution un terrain privilégié pour la confection de paradigmes et le pôle d'attraction de différentes formes de déterminisme. Pour réagir donc à un type d'histoire politique qui attribue implicitement un rôle à la Révolution avant même d'en avoir analysé à fond le fonctionnement, une autre approche a été adoptée : celle qui refuse de croire que tout était inscrit $a b$ initio dans l'événement, mais qui regarde la Révolution comme un 
phénomène original, sujet à toutes sortes de conditionnements à chaud, où rien n'était fatalement prédestiné, mais plusieurs voies étaient ouvertes : un itinéraire in fieri qui trace sa route au fur et à mesure, expérimentalement ${ }^{3}$.

L'antidote que notre étude s'est donné, vis-à-vis d'un excessif empiétement des a priori dogmatiques et des formes les plus obstinées du déterminisme, a été le choix d'opérer une suspension provisoire de jugement sur les grandes questions historiographiques : une sorte d'époké, pour emprunter une expression des philosophes sceptiques ou, si l'on préfère, de « réduction phénoménologique » en retenant la formule husserlienne. Cette opération provisoire de suspension, de mise entre parenthèses, prétend revenir aux phénomènes dans leur manifestation originelle, autant que possible non encore élaborée sous forme conceptuelle, privilégiant ainsi le flux de l'expérience vécue (Erlebnisstrom) par rapport à un emploi de catégories idéologiques trop contraignantes. Dissoudre donc une grille interprétative excessivement rigide pour saisir le déroulement de l'expérience révolutionnaire et en cerner avec un esprit plus « laïque » les mécanismes de fonctionnement. Certes il ne s'agit pas d'un refus systématique, mais, encore une fois, d'un expédient herméneutique provisoire, fonctionnel à libérer la recherche de certains conditionnements trop marqués; ceci n'empêche certainement pas pour autant de récupérer des catégories synthétiques, le débat sur les interprétations et un certain niveau d'abstraction; mais sans prétendre insérer précocement l'expérience révolutionnaire dans un lit de Procuste conceptuel trop restreint. Cette procédure d'attente nous permet en revanche d'observer les phénomènes se déployer dans toute leur trajectoire avant d'en faire des pièces ou des outils d'un «lego " politologique; il s'agit donc d'une analyse à caractère génétique, dans le but de saisir ce que je serais tenté d'appeler, en réadaptant la célèbre formule d'E.P. Thompson, the making d'une révolution.

De ce point de vue il est possible d'essayer de comprendre le fonctionnement de l'engrenage révolutionnaire sans le conditionnement des historiographies militantes cet engrenage en effet n'est pas un mécanisme inexorable, une « machine »- comme souvent on le prétend - , mais un phénomène avec ses modalités et ses inerties spécifiques que la petite échelle d'un quartier permet de bien explorer. En quittant le centre de la ville et les principaux théâtres de la vie politique parlementaire pour pénétrer dans le microcosme d'une réalité périphérique, j'ai même acquis une attitude différente et un intérêt plus circonstancié il n'était plus question de me tourner vers le faubourg Saint-Marcel dans la simple quête d'éléments destinés à enrichir l'histoire générale de la ville, en délaissant tout ce qui ne visait pas directement ce but. J'ai essayé au contraire de reconstruire une réalité munie de sa propre personnalité et d'un rythme spécifique de pulsation, non nécessairement à l'unisson avec le reste de Paris. D'où l'artifice de recommencer à raconter la Révolution du commencement à partir du point d'observation spécifique d'un quartier, en faisant partiellement abstraction de son histoire générale, tenue somme toute pour acquise. Il s'agit donc d'une démarche délibérément elliptique qui se reflète aussi sur le choix d'une bibliographie dépouillée et sélective.

Si l'approche que j'ai choisie est consciemment minimaliste et évite toute extrapolation hâtive, privilégiant le large assemblage de sources, je n'ai pas pour autant ignoré les questions de fond; celles-ci apparaissent en filigrane tout au long de la thèse. À certaines de ces questions j'ai essayé de donner une réponse pour certaines autres, j'ai avancé des hypothèses que des recherches à venir vont confirmer ou démentir. 
15 Paris devient en effet, sous la Révolution, la plus grande arène de discours et de débat politique à tous les niveaux le fossé entre la ville « qui parle » et celle « du silence » est ici bien plus restreint qu'ailleurs, car, ne serait-ce que par émulation, ceux qui veulent prendre la parole - chacun à sa façon - sont nombreux : voilà donc que nous est livré un laboratoire d'observation extraordinairement riche et varié. À l'échelle d'un quartier il a été possible donc d'examiner au microscope une large gamme de phénomènes et de comportements politiques leur origine, leur diffusion, leur standardisation. C'est le cas, par exemple, de l'expérience de la participation à la vie publique et de la pratique de la démocratie ; j'ai suivi pas à pas la mise en place d'une nouvelle sociabilité politique avec ses organismes, ses règles et ses pratiques mûries à chaud, expérimentalement; mais aussi ses inconvénients, sa crise et son déclin. J'ai pu voir apparaître et se développer le militantisme, en particulier le militantisme populaire, un phénomène nouveau, nullement prévu et qui n'attend pas l'an II pour se manifester. De même, j'ai pu suivre de près le mode de fonctionnement de l'appareil institutionnel et administratif parisien, dans le complexe jeu d'interaction de ses nombreux organismes sectionnaires.

16 Il y a, d'autre part, des concepts couramment employés par l'histoire politique et qu'on considère comme acquis dont cependant ne sont pas toujours clairs ni l'origine, ni l'évolution. Dans le cas, par exemple, du concept d'opinion publique - une abstraction de large emploi - il est particulièrement important de comprendre comment elle se forme, à quels conditionnements elle est soumise et comment concrètement elle se manifeste. Une opinion publique révolutionnaire n'apparaît pas toute prête, mais, encore une fois, est le résultat d'un processus graduel dont on peut, au niveau d'un échantillon restreint, bien cerner les étapes sous la poussée de certains événements ou de certaines réactions.

17 Curieusement, l'idée d'une évolution continue de l'Ancien Régime à la Révolution est d'habitude acceptée; on admet en effet que certaines notions ou pratiques politiques puissent avoir une gestation et même des précédents illustres bien avant la Révolution ce qu'on accepte moins souvent est en revanche que ces mêmes pratiques connaissent une évolution significative dans le temps court du processus révolutionnaire lui-même, grâce à une accélération de la conjoncture et à une intensification de la pratique politique. En fait, c'est comme si on pouvait paradoxalement cerner un temps long dans la courte durée.

18 Il en est de même en ce qui concerne le grand thème de la radicalité comme j'ai essayé de le démontrer, il ne s'agit pas d'un péché originel, inné a priori dans la Révolution, mais d'un processus qu'on peut observer et analyser, dont les mécanismes, le rythme et les accélérations répondent à une logique qu'il est important de cerner dans ses manifestations concrètes. D'ailleurs la radicalisation n'est pas une prérogative de la Terreur, au contraire elle la précède et s'étale tout au long de la période révolutionnaire débouchant certes sur le paroxysme de l'an II, mais par un itinéraire complexe entamé précocement et fait de phénomènes en chaîne. Toutefois la Terreur aussi a une histoire dans le temps court et connaît une incubation spécifique il y a une dialectique entre radicalité et Terreur qui s'exprime tant dans les hauts lieux de la politique que dans les comportements collectifs et qu'on peut analyser au ralenti, sur le plan local.

19 Sur l'ensemble de ces thèmes, qui sont au cœur de l'histoire politique de la Révolution, on ne trouvera pas dans ma thèse une interprétation conclusive, tant s'en faut, mais un 
banc d'observation approfondie et une vaste anthologie de matériaux sélectionnés, dégrossis et assemblés à la lumière justement des questions qu'on vient d'évoquer et qui peuvent servir comme point de comparaison pour d'autres recherches spécifiques ou pour des travaux de synthèse.

Même en ce qui concerne plus spécifiquement le quartier que nous présentons, on peut saisir une séquence dynamique, notamment dans la constitution d'une nouvelle identité qui change et évolue au fil des années révolutionnaires. D'une part il est question de l'image que le faubourg Saint-Marcel se fait de lui-même, mais aussi bien de celle qu'il donne de soi au reste de la ville; dans ce processus sont concernés non seulement les lieux de la politique ou la rue avec ses remous, mais sont en jeu toute la société faubourienne et le rapport qu'elle instaure avec la Révolution. C'est progressivement, quoique irrégulièrement, que cette société se refaçonne: de nouvelles valeurs se superposent aux anciennes dans les domaines les plus divers dans ce cadre mûrissent les stratégies que les différents acteurs sociaux et politiques élaborent par rapport à l'évolution de la conjoncture révolutionnaire dans son ensemble.

21 Si l'image du quartier qui ressort de notre ouvrage ne comporte aucune "révision » particulière, ni prétend non plus bouleverser immédiatement le cadre que nous livre l'historiographie parisienne traditionnelle, notre effort a été celui de comprendre, à partir de cet échantillon, comment la Révolution travaille concrètement : pour le saisir, il a fallu accepter de rentrer entre les plis de la vie faubourienne afin d'observer sur ce terrain les divers comportements politiques et, par ce biais, voir confirmées ou démenties certaines interprétations générales. À ce niveau, en effet, on peut vérifier et saisir dans le vif les étapes d'une acculturation politique, en passant par le repérage des premiers lieux d'apprentissage et de politisation, pour aboutir aux formes de sociabilité et de militantisme plus mûres et achevées.

Certes, le procédé que nous avons choisi comporte un tribut important à payer à l'érudition l'approche monographique impose en effet de reconstruire avec autant de soin que possible la trame des événements, quoique ce travail n'ajoute pas forcément des éléments nouveaux à la compréhension d'ensemble. Néanmoins, malgré les inévitables lourdeurs que cela représente, je n'ai pas cru devoir reculer face à cette tâche, dans le souci de compléter le dossier et par cohérence avec une étude de cas qui, pour se prétendre exhaustive et démontrer toutes ses potentialités, n'admît point de raccourcis. D'ailleurs, en ce qui concerne par exemple les journées insurrectionnelles, nous ne disposons pas d'analyses spécifiques sur le rôle joué par les différents quartiers; toute tatillonne que cette démarche puisse paraître, il ne faut pas y renoncer, car elle est en mesure de nous fournir des éléments utiles sur les comportements de masse et les dynamiques insurrectionnelles. C'est sur ce terrain par exemple que se noue le complexe rapport entre spontanéité et direction politique dont le rôle respectif demeure très important à éclaircir de même c'est dans l'insurrection que se développent à chaud certains facteurs de radicalisation souvent imprévus qu'il est important de saisir car, loin de disparaître avec la fin de l'événement, ils constituent des précédents qui s'inscrivent durablement dans l'expérience révolutionnaire jusqu'à en conditionner les suites.

23 Notre histoire du faubourg Saint-Marcel ne se réduit pas pour autant à une suite d'événements des noyaux thématiques particulièrement importants dans la vie du quartier - et parfois très bien documentés - ont été cernés et suivis tout au long des 
années révolutionnaires. C'est le cas, par exemple, des élites locales dont on a essayé de reconstruire le parcours entre Ancien Régime et Révolution, non seulement dans le cadre d'une étude sur la notabilité, mais aussi à travers une analyse des comportements politiques. Certaines trajectoires typiques ont été esquissées aussi bien que certains tempéraments, avec le soin toutefois de ne pas les extraire du contexte qui les avait produits. Parmi ces élites, les intellectuels, présents en grand nombre grâce aux différents établissements scientifiques et universitaires installés dans cette partie de la capitale, jouent un rôle tout à fait particulier ; le rapport contradictoire qu'ils nouent avec la politique révolutionnaire est un thème qui mérite toute notre attention.

$\mathrm{Au}$ niveau des couches populaires, une attention constante a été consacrée aux problèmes relevant du monde du travail et de l'assistance : un sujet de très grande importance, notamment dans un quartier populaire, un sujet souvent sous-estimé au bénéfice de l'étude sur l'engagement politique. Cette attention, qui s'étale sur toute la période révolutionnaire, permet de vérifier concrètement dans ce milieu tout à fait particulier les éléments de continuité et de rupture avec le passé et la façon où monde $\mathrm{du}$ travail et politique interagissent. Il en est de même en ce qui concerne la communauté locale de quartier et ses formes de sociabilité dont on connaît le poids dans le Paris d'Ancien Régime ; même si elle est soumise à un processus d'érosion, dans la seconde moitié du xviIIe siècle, il n'en reste pas moins important d'aller voir ce que de cette expérience de longue durée réapparaît dans la nouvelle koiné révolutionnaire après que la vie populaire est passée à travers le philtre d'une politisation intense.

Il n'y a dans ce qui précède qu'un aperçu des questions que je me suis posées; la réponse dépendant à l'évidence largement de l'état des sources, ceci explique certaines incohérences dans la façon dont ces mêmes thèmes sont affrontés tout au long de la thèse. Il se peut que d'autres quartiers de Paris disposent d'un dossier documentaire différemment composé, ce qui permettrait de mieux développer certains aspects restés insuffisamment traités dans le cadre du faubourg Saint-Marcel. C'est pourquoi il vaudrait la peine d'appliquer ces mêmes critères de recherche à d'autres lieux de la ville, ne serait-ce que dans le cadre d'un travail comparatif. Dans cette perspective, je crois avoir proposé une grille de recherche et un type de travail sur les sources qui peuvent contribuer à rouvrir un chantier, celui de l'histoire parisienne sous la Révolution, somme toute délaissé, mais encore riche de potentialités.

\section{NOTES}

1.Doctorat d'État ès lettres et sciences humaines sous la direction de Michel Vovelle, Université de Paris I Panthéon-Sorbonne. Jury : M. Maurice Agulhon (président), Mme Catherine Duprat, MM.Colin Lucas, Jacques Revel, Daniel Roche, Michel Vovelle (3 novembre 1999).

2.H. BURSTIN, Le faubourg Saint-Marcel à l'époque révolutionnaire. Structure économique et composition sociale, Paris, Société des études robespierristes, 1983. 
3.Un exemple récent particulièrement significatif de cette tendance nous est fourni par T.TACKETT, Becoming a revolutionary. The deputies of the French National Assembly and the emergence of a revolutionary culture (1789-1790), Princeton, Princeton University Press, 1996. 